Article

\title{
Bias-Modulated High Photoelectric Response of Graphene-Nanocrystallite Embedded Carbon Film Coated on n-Silicon
}

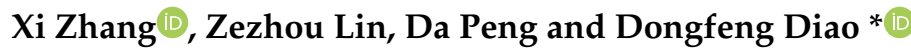

Institute of Nanosurface Science and Engineering, Guangdong Provincial Key Laboratory of Micro/Nano Optomechatronics Engineering, Shenzhen University, Shenzhen 518060, China; zh0005xi@szu.edu.cn (X.Z.); zezhoulin@163.com (Z.L.); pandavmac@163.com (D.P.)

* Correspondence: dfdiao@szu.edu.cn; Tel.: +86-755-26902415

Received: 25 January 2019; Accepted: 20 February 2019; Published: 1 March 2019

\begin{abstract}
We propose that bias-modulated graphene-nanocrystallites (GNs) grown vertically can enhance the photoelectric property of carbon film coated on n-Si substrate. In this work, GN-embedded carbon (GNEC) films were deposited by the electron cyclotron resonance (ECR) sputtering technique. Under a reverse diode bias which lifts the Dirac point of GNs to a higher value, the GNEC film/n-Si device achieved a high photocurrent responsivity of $0.35 \mathrm{~A} / \mathrm{W}$. The bias-modulated position of the Dirac point resulted in a tunable ON/OFF ratio and a variable spectral response peak. Moreover, due to the standing structured GNs keeping the transport channels, a response time of $2.2 \mu \mathrm{s}$ was achieved. This work sheds light on the bias-control wavelength-sensitive photodetector applications.
\end{abstract}

Keywords: graphene nanocrystallite; bias-modulated Fermi level; carbon film; photoelectric response; tunable spectral response

\section{Introduction}

Photoelectric sensors are widely used in optical communication, infrared ranging, environmental monitoring, military security inspection, biomedical imaging, and scientific research [1-4]. Graphene is a promising candidate for new-generation photodetectors due to its outstanding photoelectric properties $[5,6]$. The combination of graphene and silicon, with their known conductive behaviors, offers new unique properties and draws great research attention $[7,8]$. However, plane-graphene photodetectors present low photocurrent responsivity due to the lack of trapping centers in plane-graphene, which causes the rapid recombination of photoexcited carriers. Large-scale graphene with few defects is difficult to obtain using modern techniques such as chemical vapor deposition (CVD) [9-14], reduced graphene oxide (RGO) [15], and mechanical lift-off techniques [16]. The effective areas of the reported graphene/silicon photodetector are usually tens of $\mu \mathrm{m}^{2}$. Moreover, the transfer technique for the graphene/silicon heterojunction is complicated and limits the material's industrial applications. Therefore, a method to mass-produce a photodiode with low cost and high responsivity is highly desired.

Amorphous carbon (a-C) thin films were synthesized from coal as a solid carbon source [17]. The a-C thin film has an optical transmittance of $>96 \%$ in the spectral range from $350 \mathrm{~nm}$ to $900 \mathrm{~nm}$, and it can be transferred to various substrates. In 1996, Yu prepared a carbonaceous thin-film/n-type silicon layer photovoltaic cell. It was confirmed that the carbonaceous film/n-type silicon junction was a heterojunction [18]. The a-C film is the earliest carbon-based material applied in heterojunctions for photoelectric devices with a responsivity of $0.18 \mathrm{~A} / \mathrm{W}$ [19]. In 2012, Xue et al. tested the substrate 
resistivity [20], light-induced resistance effect [21], and photoconductivity [22] of Pd-doped amorphous carbon film $/ \mathrm{SiO}_{2} / \mathrm{Si}$ heterojunctions. The device size of the a-C film photodetector can be as large as a commercial photodetector ( tens of $\mathrm{mm}^{2}$ ). However, the responsivity of a-C film is still rather low.

Recently, we proposed a method for the direct preparation of graphene nanocrystallite-embedded carbon (GNEC) film via a low energy electron irradiation technique [23]. Unlike a-C film, GNEC film contains many vertically grown graphene nanocrystallites (GNs). It exhibits a good magnetic and triboelectric performance $[23,24]$ due to the large amount of graphene edges in GNEC film, which serve as trapping centers for electrons and net spin. Hence, it is expected that GNEC film can be applied in a photoelectric response area, since the edge electron trapping centers may assist with photocurrent generation in the photovoltaic process.

In this paper, we report the photo-electric behavior of GNEC films coated on n-Si substrates prepared by electron cyclotron resonance (ECR) plasma sputtering technology under bias voltage modulation. The external bias could modulate the Fermi level $\left(E_{\mathrm{F}}\right)$ of GNs and help to form a $\mathrm{p}-\mathrm{n}$ junction with n-Si. The photoelectric properties of the GNEC film/n-Si heterojunction were tested, and the mechanism of the nanostructure's effect on the photoelectric properties of the GNEC coating was unveiled.

\section{Experimental Methods}

The GNEC films were coated on n-Si using an ECR plasma sputtering system. A detailed description of the sputtering system was reported in our previous works. A 500-W microwave was delivered into the vacuum chamber to generate the plasma at an argon pressure of $4 \times 10^{-2} \mathrm{~Pa}$. The mirror confinement magnetic field was applied in order to enhance the plasma density. A negatively biased glassy carbon target is known to attract $\mathrm{Ar}^{+}$ions to sputter carbon species towards the substrate [25]. The substrate was a single-sided polished 4-inch N-type conductive silicon wafer (square resistance of $\sim 10 \Omega, 0.5 \mathrm{~mm}$ thickness). A deposition time of $30 \mathrm{~min}$ was adopted in this work to control the thickness to be around $70 \mathrm{~nm}$. A positive deposition bias $\left(V_{\text {dep }}\right)$ was applied on the substrate to attract electrons [26]. With the assistance of the low-energy electrons, GNs grow perpendicularly to the substrate. We prepared films under $V_{\text {dep }}=20,50$, and $80 \mathrm{~V}$. The nanostructures of the carbon films were analyzed using Raman spectroscopy (HORIBA, HR-Resolution; wavelength of $532 \mathrm{~nm}$ ) and transmission electron microscopy (TEM, JEOL, JEM-3200FS).

A finger-type 50-nm-thick gold electrode was deposited on top of the GNEC film using UV lithography [27]. A 50-nm-thick flat gold electrode was deposited on the back of the silicon substrate. The Lakeshore TTPX cryogenic probe station covered by a built-up darkroom was used as the electrode contact. The optical setup was also placed in the built-up darkroom. Free-space lasers were used as the light source with a spot size of approximately $0.2 \mathrm{~mm}^{2}$. A Keithley 4200 -scs semiconductor characterization analyzer was used to measure the I-V curves. To measure the frequency response, a diode laser (Coherent OBIS, $\lambda=785 \mathrm{~nm}$ ) was modulated ON/OFF with a $50 \%$ duty cycle by a $100-\mathrm{kHz}$ square waveform generated by a function generator (Keysight 33,600 A). The rise/fall time of the modulated diode laser was $10 \mathrm{~ns}$. The spectral response was measured by a white light source, a monochromator, a chopper, and a lock-in amplifier. A xenon light in the visible to near-infrared $(300 \mathrm{~nm}$ to $1100 \mathrm{~nm}$ ) range was used as the white light source. The xenon lamp light was dispersed by a monochromator (precision of $0.5 \mathrm{~nm}$ ) and the average power was in the order of $10 \mu \mathrm{W} / \mathrm{nm}$.

\section{Results and Discussion}

\subsection{Nanostructure Characterization of GNEC Films}

The carbon films were coated on the n-Si substrate using an ECR sputtering system, as shown in Figure 1. The thickness of the GNEC film on n-Si was about $70 \mathrm{~nm}$, as measured by a profilometer (Bruker, Dektak-XT, AZ, USA). The coated wafer was cut into $5 \times 5 \mathrm{~mm}^{2}$ square pieces. A finger-shaped electrode was deposited on the surface of the GNEC film by UV lithography, and a 50-nm flat gold 
film was deposited on the back of the Si substrate. The finger-type mask was designed with a central spine ( 300 $\mu \mathrm{m}$ width) and two sides of branches ( 150 $\mu \mathrm{m}$ width). The spacing between the two branches was $\sim 500 \mu \mathrm{m}$. The spine and branches were all designed in the shape of wedges to collect the electrons efficiently. High-resolution transmission electron microscopy (TEM) was used to explore the cross-section sample of the 50-V film, as shown in Figure 1c. A cross-section sample with a lateral thickness of $100 \mathrm{~nm}$ was prepared by focused ion beam (FIB) after a protection layer was deposited on top (see Figure S1). The minimal thickness was more conducive to our observation of the vertical growth structure of GNs under TEM. The etching energy was high during FIB deposition, so it was necessary to deposit a $2 \mu \mathrm{m}$ protective layer (platinum material) on the surface of the sample to prevent damage to the surface morphology during the FIB etching process and TEM observation. Although an overlay image of a 100-nm lateral thickness cross-section was revealed during the TEM observation, as shown in the inset in Figure 1a, the tendency of GNs to grow perpendicular to the substrate was conspicuous.
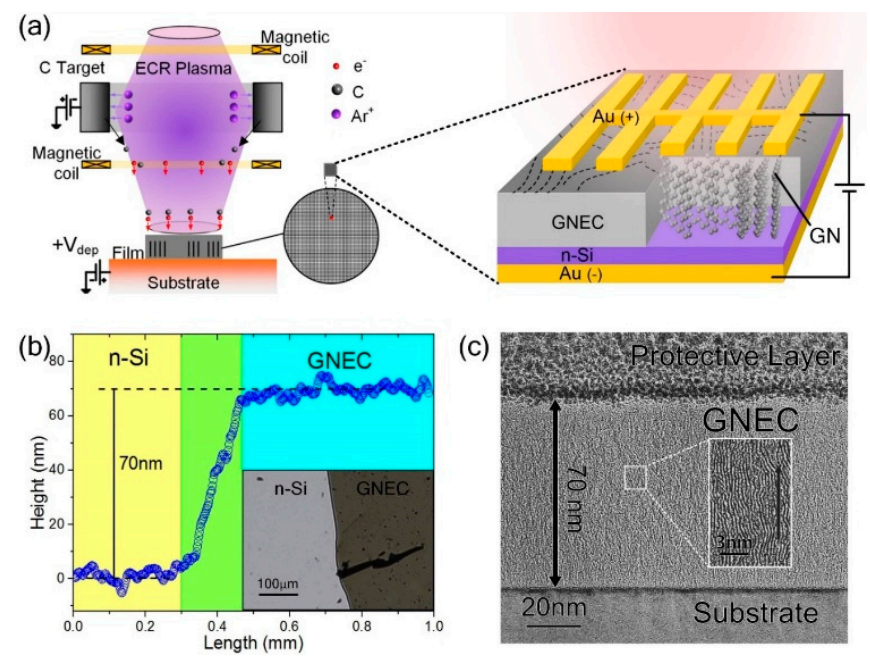

Figure 1. (a) A schematic setup of the electron cyclotron resonance (ECR) plasma low-energy electron deposition. The inset shows standing structured graphene nanocrystallite (GN) induced by low-energy electrons irradiation (modulated by $V_{\text {dep }}$ ) in graphene nanocrystallite-embedded carbon (GNEC) film. Diode bias $\left(V_{\text {diode }}\right)$ is applied on the GNEC film against Si. (b) The stepped profile of GNEC film coated on n-Si. (c) High-resolution TEM image of the cross-section sample of the $50-\mathrm{V}$ film prepared by focused ion beam (FIB) deposition.

Transmission electron microscopy (TEM) and Raman spectra of carbon films coated under ECR low-energy electron irradiation at $V_{\text {dep }}=20 \mathrm{~V}, 50 \mathrm{~V}$, and $80 \mathrm{~V}$ are shown in Figure $2 \mathrm{a}-\mathrm{c}$. The red and green boxes in Figure $2 \mathrm{a}-\mathrm{c}$ mark the areas with and without GN, respectively, and the insets are their fast Fourier transform (FFT) images [28,29]. The FFT images demonstrate that inside the GN two Laue spots appear, corresponding to the $(0001)^{*}$ facet of the multilayer graphene [30]. The reciprocal lattice distance is measured as $\mathrm{d}_{(0001)}^{*}=5.98 / 2 \mathrm{~nm}^{-1}=2.99 \mathrm{~nm}^{-1}$. Thus, the interplanar distance can be calculated as $\mathrm{d}=1 / 2.99 \mathrm{~nm}=0.334 \mathrm{~nm}$, in accordance with the (0001) facet distance of multilayer graphene. In the green square area, the FFT image does not show a pattern, indicating no GN formation. In the TEM image, we used white circles to indicate the boundaries of the GN on the GNEC film. The average size of the white circles indicates the width of the GN boundary. At $V_{\text {dep }}=20 \mathrm{~V}$, the carbon film has an a-C structure. At $V_{\text {dep }}=50 \mathrm{~V}$, the average size of the GN boundary is $\sim 4 \mathrm{~nm}$. At $V_{\mathrm{dep}}=80 \mathrm{~V}$, the average size of the $\mathrm{GN}$ boundary is increased to $\sim 15 \mathrm{~nm}$. 
(a)
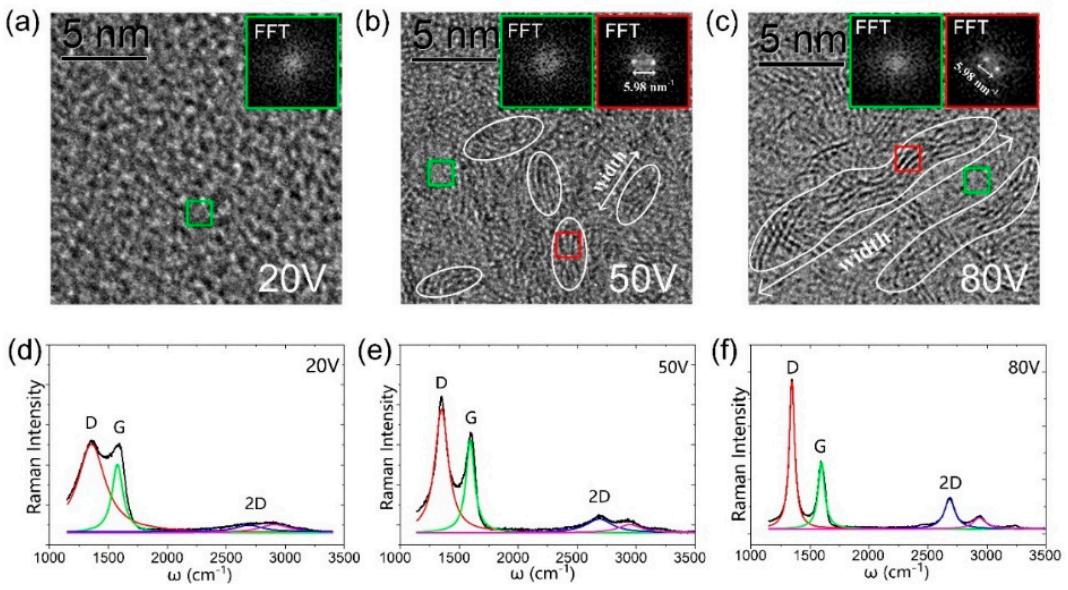

Figure 2. High-resolution TEM plan-view images of GNEC films deposited at a $V_{\text {dep }}$ of (a) $20 \mathrm{~V}$, (b) 50 $\mathrm{V}$, and (c) $80 \mathrm{~V}$. Insets are the fast Fourier transform (FFT) images of the selected region (green and red squares). As the $V_{\text {dep }}$ increases, the GN boundary width increases. Raman spectra of GNEC films with a $V_{\text {dep }}$ of (d) $20 \mathrm{~V}$, (e) $50 \mathrm{~V}$, and (f) $80 \mathrm{~V}$. As the $V_{\text {dep }}$ increases, D, G, and 2D peaks are all sharpened.

Raman spectra of films at $V_{\mathrm{dep}}=20 \mathrm{~V}, 50 \mathrm{~V}$, and $80 \mathrm{~V}$ are shown in Figure 2d-f. D, G, and 2D peaks were decomposed by Lorentz function fitting [31-34]. At $V_{\text {dep }}=20 \mathrm{~V}$, the D, G, and 2D profiles are vague and wide, and the intensity ratio between $D$ and $G$ peaks $\left(I_{D} / I_{G}\right)$ is almost 1 , indicating the formation of a-C structures. At $V_{\text {dep }}=50 \mathrm{~V}$, a stronger 2D peak around $2700 \mathrm{~cm}^{-1}$ appears and the $\mathrm{I}_{\mathrm{D}} / \mathrm{I}_{\mathrm{G}}$ increases to 1.34 , indicating the formation of small-size GNs in the carbon film. At $V_{\text {dep }}=80 \mathrm{~V}$, a sharp $2 \mathrm{D}$ peak can be observed and $\mathrm{I}_{\mathrm{D}} / \mathrm{I}_{\mathrm{G}}$ increases to 2.25. This indicates that, with the increase of low-energy electron radiation energy, the structure of GN on GNEC film tends to be ordered and the width of the GN boundary tends to increase.

Figure $2 \mathrm{~d}-\mathrm{f}$ also show the red shift of the 2D peak position versus $V_{\text {dep }} . \omega$ decreases from 2693 to $2688 \mathrm{~cm}^{-1}$ and then to $2683 \mathrm{~cm}^{-1}$ as $V_{d e p}$ increases from 20 to $50 \mathrm{~V}$ and then to $80 \mathrm{~V}$. The shift of the 2D band can be explained by the edge quantum effect theory $[35,36]$. This theory clarifies the correlation, $\Delta \omega \infty\left(E_{K} / \mu\right)^{1 / 2} /\left(d_{k} K\right)$, among the shift of the 2D peak $(\Delta \omega)$ from the $\omega$ (Bulk) of large graphene bulk to the nanocrystallite $\omega(K)$, the size $K$, the average bond length $\left(d_{K}\right)$, the average bond energy $\left(E_{K}\right)$, and the reduced mass of the dimer atoms $(\mu)$. Thus, as the crystallite size increases, the average bond length expands and the average bond energy drops, leading to the decrease of $\omega$ towards the reference point of large graphene. Thus, the trend of the red shift of $\omega(K)$ as $V_{\text {dep }}$ increases agrees well with the theoretical prediction, verifying the increase of the nanocrystallite size of GNEC film.

\subsection{Device Characterization and Nanostructure-Dependent Photocurrent}

$V$ curves in darkness and at a power $(\mathrm{P})$ of 10 or $40 \mathrm{~mW}$ at a wavelength of $785 \mathrm{~nm}$ laser are shown in Figure 3a-c. The dark currents showed rectification characteristics. Since the film with $V_{\text {dep }}=20 \mathrm{~V}$ had an amorphous structure, a low photocurrent was generated under reverse bias. At a diode bias of $-5 \mathrm{~V}$, the GNEC film at $V_{\text {dep }}=50 \mathrm{~V}$ produced a larger photocurrent of $9 \times 10^{-3} \mathrm{~A}$. As the GN size increased, the GNEC film at $V_{\text {dep }}=80 \mathrm{~V}$ produced the highest photocurrent of $1.44 \times 10^{-2} \mathrm{~A}$ at reverse $V_{\text {diode }}=-5 \mathrm{~V}$. Increasing the irradiation voltage $\left(V_{\text {dep }}\right)$ enhanced the crystallization of the GNEC film, leading to a larger photocurrent under reverse bias. Photocurrents are extremely low at zero bias and increase drastically with reverse bias. Graphene/n-Si [37] also exhibited a drastically photocurrent depression at $V_{\text {diode }}=0 \mathrm{~V}$ due to the Dirac cone of graphene. This behavior at zero bias is largely different from that of the GNEC/p-Si device [38]. The GNEC film/p-Si showed a considerably large photocurrent at $V_{\text {diode }}=0 \mathrm{~V}$ due to the natural $\mathrm{p}-\mathrm{n}$ junction formation between the electron-trapped GNs and p-Si. In contrast, in GNEC/n-Si, the reverse bias could depress the GNs' Fermi level to open up available energy states for holes to inject. Under illumination, the flux of photocurrent was limited by the density of states of graphene nanocrystallites near the Fermi level. Thus, the minimum 
photocurrent points in Figure 3 roughly indicate the location of the minimum density of states points. It decreased from -0.12 to $-0.17 \mathrm{~V}$ and further to $-0.2 \mathrm{~V}$ as the $\mathrm{V}_{\text {dep }}$ increased from 20 to $50 \mathrm{~V}$ and finally to $80 \mathrm{~V}$ (see Figure S2). This is because the more crystallized $80 \mathrm{~V}$ film formed a stronger $\mathrm{p}-\mathrm{n}$ junction with $n-S i$ and the relative position of $E_{F}$ had a deeper location. A stronger photocurrent under illumination was generated under reverse bias. Photocurrents almost reached saturation at $V_{\text {diode }}=-2 \sim-3 \mathrm{~V}$. As the illumination power decreased from 40 to $10 \mathrm{~mW}$ the saturation voltage decreased, since weak light can be easily used by the device while reverse bias cannot make out a further product. The diode bias-induced tunability of the relative positions of the Fermi levels resulted in a tunable $\mathrm{ON} / \mathrm{OFF}$ ratio. The $\mathrm{ON} / \mathrm{OFF}$ ratio of the $80 \mathrm{~V}$ film device varied from 3.2 at $-0.2 \mathrm{~V}$ to 21.4 at $-2 \mathrm{~V}$. The application of a reverse $V_{\text {diode }}$ raised the Fermi level $\left(\mathrm{E}_{\mathrm{f}}\right)$ of $\mathrm{GN}$, inducing the p-type behavior of GN and forming a larger junction with $\mathrm{n}-\mathrm{Si}$.
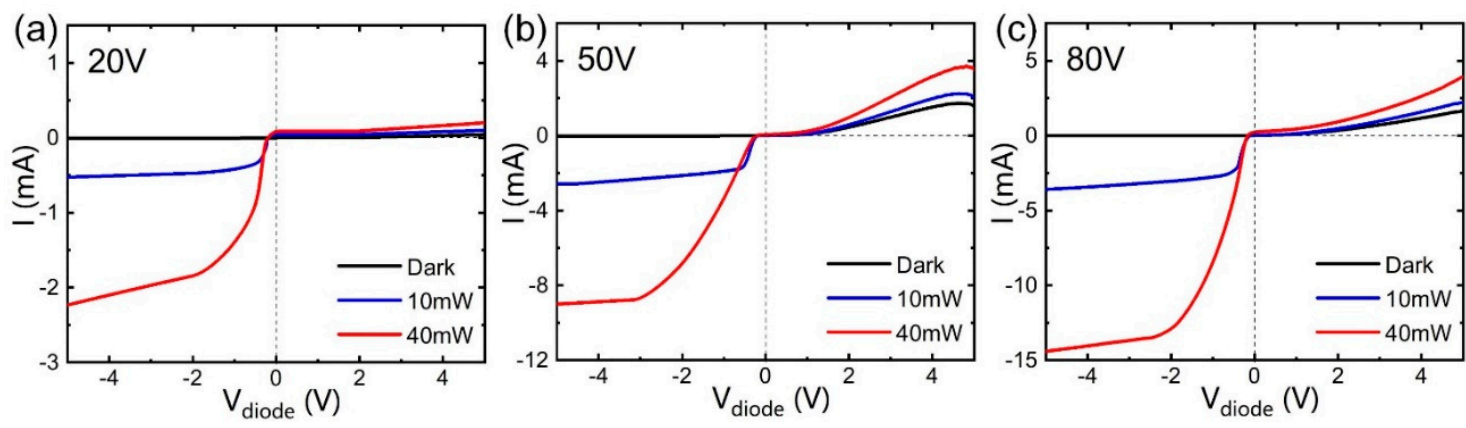

Figure 3. Current-voltage (I- $\left.V_{\text {diode }}\right)$ curves under darkness and illumination $(\mathrm{P}=10$ or $40 \mathrm{~mW})$ of $785 \mathrm{~nm}$ laser of carbon films/n-Si with a $V_{\text {dep }}$ at (a) $20 \mathrm{~V}$, (b) $50 \mathrm{~V}$, and (c) $80 \mathrm{~V}$. The GNEC film/n-Si exhibits a large photocurrent under reverse $V_{\text {diode. }}$. As the reverse bias voltage increases from 0 to $-2 \sim-3 \mathrm{~V}$, the photocurrent tends to saturate.

\subsection{Nanostructure-Dependent Spectral Responsivity}

As shown in Figure 4, the spectral responsivity curves of the 50 and 80 V GNEC film devices at different values of $\mathrm{V}_{\text {diode }}$ were obtained. The GNEC/n-Si showed a broadband photo-detection ability and the peak position was located at the near-infrared region. The $80 \mathrm{~V}$ GNEC film showed a higher responsivity of $0.35 \mathrm{~A} / \mathrm{W}$ compared to that of the $50 \mathrm{~V}$ GNEC film $(0.27 \mathrm{~A} / \mathrm{W})$ under a $900 \mathrm{~nm}$ laser, due to the increasing crystallization of GNs in the $80 \mathrm{~V}$ film. Since the illumination power of the white light source was rather weak $(\sim 10 \mu \mathrm{W} / \mathrm{nm})$, the $\mathrm{R}$ difference between -1 and $-2 \mathrm{~V}$ was not as large as that in Figure 3. This is because the saturation voltage is quite low under weak light. As the bias decreased to $0 \mathrm{~V}$, the peak responsivities decreased to low values $(2.42 \mathrm{~mA} / \mathrm{W}$ for the $50 \mathrm{~V}$ film and $0.12 \mathrm{~A} / \mathrm{W}$ for the $80 \mathrm{~V}$ film). It can also be seen that the peak position blue-shifted with reverse bias, increasing from 0 to $-2 \mathrm{~V}$ (from 1035 to $900 \mathrm{~nm}$ of the $80 \mathrm{~V}$ film). This is because the applied reverse bias can raise the Dirac point of the GN to a higher value, and increase the potential barrier of the $\mathrm{p}-\mathrm{n}$ junction interface. Thus, the electron excitation requires a larger photon energy, leading to the blue-shift phenomenon. The bias-dependent response peak not only confirms the bias-modulation to the Dirac point of the GNs, but also sheds light on the tunable spectral sensitivity applications of GNEC/n-Si devices. 

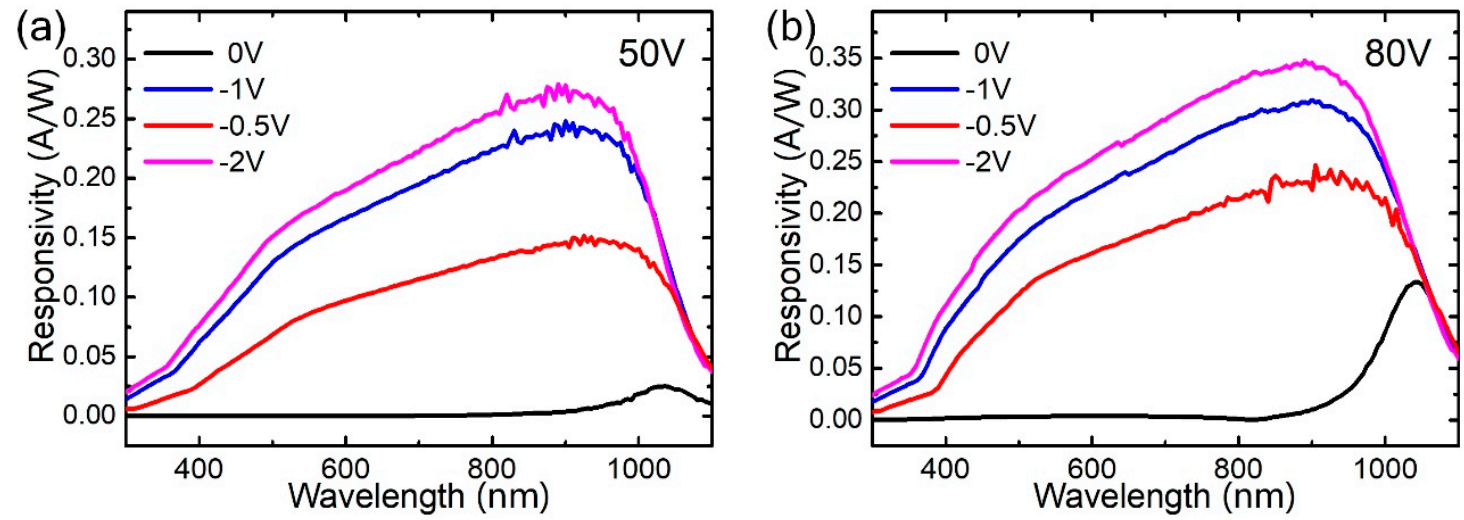

Figure 4. (a) The variation of the photocurrent responsivity $\left(V_{\text {dep }}=50 \mathrm{~V}\right)$ in the wavelength range from 300 to $1100 \mathrm{~nm}$ under different bias voltages $\left(V_{\text {diode }}=0 \mathrm{~V},-0.5 \mathrm{~V},-1 \mathrm{~V},-2 \mathrm{~V}\right)$. (b) The variation of the photocurrent responsivity $\left(V_{\text {dep }}=80 \mathrm{~V}\right)$ in the wavelength range from 300 to $1100 \mathrm{~nm}$ under different bias voltages $\left(V_{\text {diode }}=0 \mathrm{~V},-0.5 \mathrm{~V},-1 \mathrm{~V},-2 \mathrm{~V}\right)$. Applying a reverse bias voltage will cause a blue shift in the response peak.

\subsection{Response Time}

Generally, the increase in light responsivity is accompanied by an increase in the light response time [39]. Unlike other materials, a large number of GNs on GNEC membranes play a crucial role in charge transport. GN grows vertically on a substrate, increasing the charge transport channels, thereby increasing the light response speed. As shown in Figure 5a,b, the optical response speed of the GNEC film device was tested under square wave frequencies of 1 and $100 \mathrm{kHz}$. The response time of the GNEC film/n-Si device was $2.2 \mu$ s, enabling commercial fast-response applications such as high-speed imaging, barcode scanners, and photoelectric encoders. In addition, the GNEC film/n-Si device exhibited a recovery time of $2.7 \mu \mathrm{s}$, owing to the fast carrier diffusion time induced by the electron doping during GNEC film deposition.
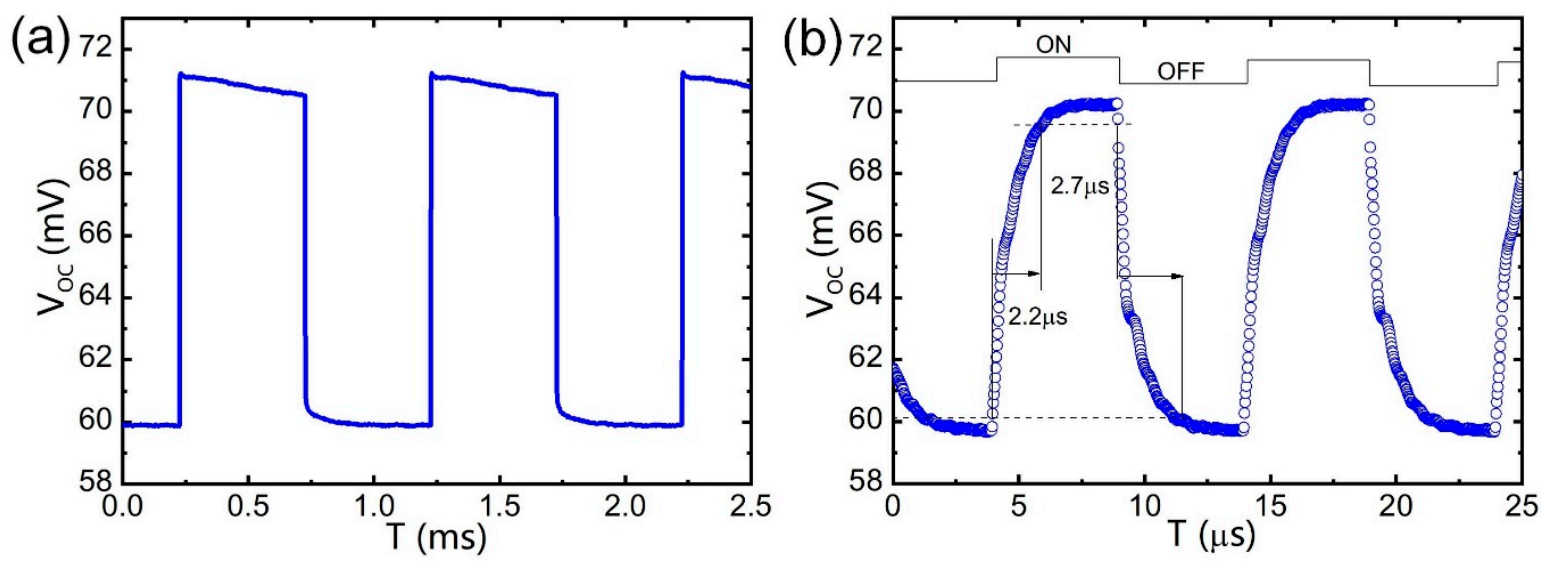

Figure 5. (a) Response time of the GNEC film/n-Si photodetector under $1 \mathrm{kHz}$ square wave optical signal. (b) Response time of the GNEC film/n-Si photodetector under $100 \mathrm{kHz}$ square wave optical signal. High sensitivity is demonstrated, the ON/OFF response rise time is $2.2 \mu$ s, and the recovery time is $2.7 \mu \mathrm{s}$.

In contrast to plane-graphene, GNEC film is a directly deposited carbon film which contains a large amount of standing structured GNs. Although plane-graphene has massless Dirac Fermions and presents an ultrafast response [40], the device area is usually limited to tens of $\mu \mathrm{m}^{2}$ and a complicated transfer technique is needed. In contrast, the a-C film device area is usually tens of $\mathrm{mm}^{2}$ and no transfer is needed for industrial applications. We aim to enhance the photoresponsivity of such 
devices by introducing standing structured GNs into the film. Despite its large area of $5 \times 5 \mathrm{~mm}^{2}$, the performance of our device was outstanding compared with some other small-area plane-graphene devices. In comparison with the previously reported graphene photodetectors, we summarize some features of the previously reported photodetectors in Table 1 . The device developed here showed a high responsivity $(0.35 \mathrm{~A} / \mathrm{W})$ compared with a traditional a-C film device $(0.18 \mathrm{~A} / \mathrm{W})$. In addition, the response time was reduced to $2.2 \mu \mathrm{s}$. The edge of the nanosheet acted as electron capture centers. Under reverse bias, the device exhibited bias modulation characteristics. The GNEC film prepared by ECR technology thus has the characteristics of low cost, easy preparation, and large-area production.

Table 1. Summary of device parameters of several typical 2D material photodetectors previously reported and our own device.

\begin{tabular}{cccc}
\hline Type of Devices & Response Time & Responsivity & Wavelength \\
\hline a-C film/Si [19,24] & - & $180 \mathrm{~mA} / \mathrm{W}$ & $850 \mathrm{~nm}$ \\
MoS $_{2}-\mathrm{WS}_{2}[41]$ & $4 \mu \mathrm{s}$ & $4.36 \mathrm{~mA} / \mathrm{W}$ & $532 \mathrm{~nm}$ \\
Gr-MoS 2 -Gr [43] & $50 \mu \mathrm{s}$ & $6 \mathrm{~mA} / \mathrm{W}$ & $532 \mathrm{~nm}$ \\
Plasmon resonance Au nanoparticle-Gr [42] & $50 \mu \mathrm{s}$ & $220 \mathrm{~mA} / \mathrm{W}$ & $488 \mathrm{~nm}$ \\
Combined Monolayer Gr (MLG)/Si [44] & $100 \mu \mathrm{s}$ & $29 \mathrm{~mA} / \mathrm{W}$ & $850 \mathrm{~nm}$ \\
GNEC/n-Si (this work) & $2.2 \mu \mathrm{s}$ & $350 \mathrm{~mA} / \mathrm{W}$ & $850 \mathrm{~nm}$ \\
\hline
\end{tabular}

\subsection{Discussion of Principle}

Under the irradiation of low-energy electrons, a large amount of standing structured GNs are vertically grown to provide a high density of edges for the GNEC film. Figure 6a illustrates the energy band diagram, showing the Fermi levels of $\mathrm{GN}\left(E_{\mathrm{f}}(\mathrm{GN})\right)$ and $\mathrm{n}-\mathrm{Si}\left(E_{\mathrm{f}}(\mathrm{Si})\right)$ at thermal equilibrium in darkness. The Dirac cone shape on the left is an illustration of the band of GNs. In the film, it may not necessarily be that shape. The Dirac point represents the minimum density of states point. The edge states of GNs tend to capture electrons and raise the $E_{\mathrm{f}}(\mathrm{GN})$ [45]. The two curves on the right represent the top of the valence band $\left(E_{\mathrm{V}}\right)$ and the bottom of the conduction band $\left(E_{\mathrm{C}}\right)$ of $\mathrm{n}-\mathrm{Si}$. The $E_{\mathrm{f}}$ (Si) of n-Si locates near the valence band $\left(E_{\mathrm{V}}\right)$ and bends at the interface with a potential barrier $q \mathrm{~V}_{\mathrm{d}}$ due to the formation of a $\mathrm{p}-\mathrm{n}$ junction. As shown in Figure $6 \mathrm{~b}$, when reverse bias voltage is applied, quasi Fermi levels—separately for holes of n-Si $\left(E_{\mathrm{f}}{ }^{\prime}(\mathrm{Si})\right)$ and electrons of $\mathrm{p}-\mathrm{GN}\left(E_{\mathrm{f}}{ }^{\prime}(\mathrm{GN})\right)$-are formed in the non-equilibrium situation. An applied reverse bias raises the Dirac point of GNs, increasing the chemical potential disparity between GNs and n-Si and leading to the formation of junctions [46]. The potential barrier was increased by $q \mathrm{~V}_{\mathrm{r}}$, preventing the dark current. The applied reverse bias can raise the Dirac point of GNs to a higher value, opening up a large number of accessible states for the holes to inject into and allowing a complete collection of the injected holes. Under illumination, photo-excited holes tend to flow into the accessible GN states. Hence, the electron-hole recombination rate is drastically reduced by GN trapping centers, leading to an outstanding responsivity.

There is huge potential for GNEC film to further enhance its responsivity, for example, by increasing the density of GN edges, preventing dark current leakage by introducing an interface insulator [3], nitrogen-doping, or other dopants. Besides, increasing the substrate beyond $80 \mathrm{~V}$ would increase the crystallization of the GNs and bring the film towards a graphite-like structure. Technically, excessive substrate bias may cause anomalies in the deposition equipment. Physically, increasing the deposition bias would probably enhance the performance of the device, but there should be a critical point somewhere larger than $80 \mathrm{~V}$. At that critical point, the graphite-like film would lack trapping centers and the photoelectric performance would decrease. 

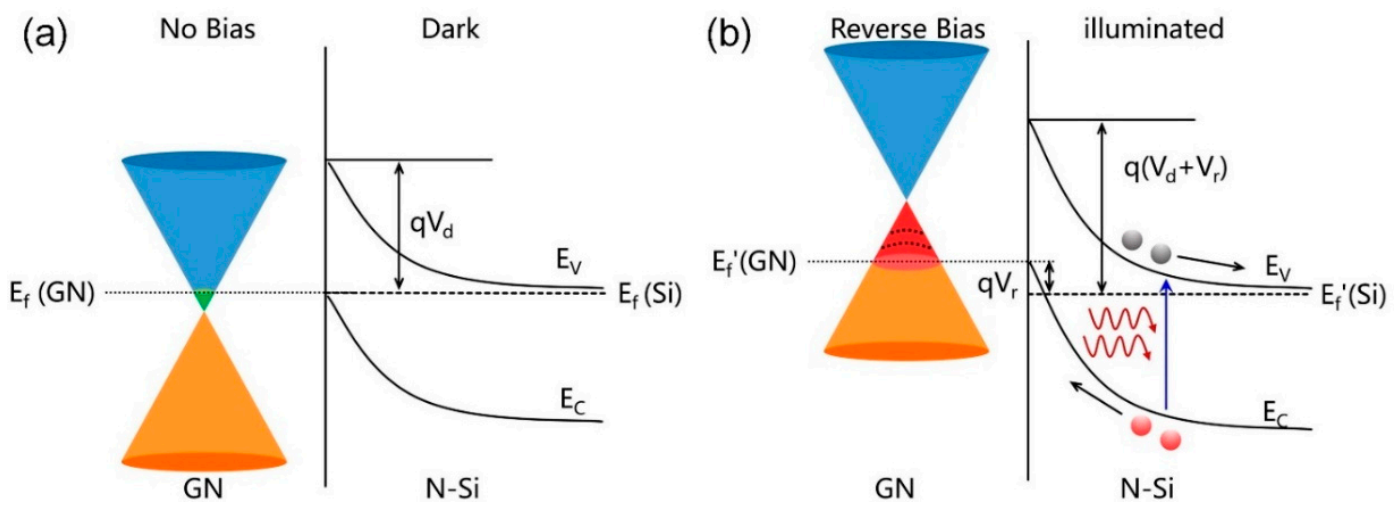

Figure 6. (a) Energy band structure diagram of the GNEC film/n-Si heterojunction at zero bias in the dark state. (b) Energy band structure diagram of the GNEC film/n-Si heterojunction under reverse bias in illumination.

\section{Conclusions}

GNs vertically grown in a GNEC film can prominently improve the photoelectric performance of the material by improving its ability to collect photogenerated electrons and prolonging the lifetime of photoexcited carriers. The GNEC film/n-Si heterojunction exhibited a high photocurrent responsivity of $0.35 \mathrm{~A} / \mathrm{W}$ under reverse bias, compared with the responsivity of $0.18 \mathrm{~A} / \mathrm{W}$ of a-C film $/ \mathrm{n}-\mathrm{Si}$. In the GNEC film preparation process, we can control the crystallinity by adjusting the energy of low-energy electron irradiation, so that the photoelectric performance of the GNEC film/n-Si photodetector can be improved.

The GNEC film coated on n-Si exhibited excellent photocurrent responsivity $(0.35 \mathrm{~A} / \mathrm{W})$ and a highly sensitive response time $\left(\tau_{\text {rise }}=2.2 \mu \mathrm{s}\right)$ under reverse bias. The excellent photoelectric performance exhibited under reverse bias was attributed to the GN crystallization and the increased Dirac cone. The bias-modulated position of the Dirac point resulted in a tunable ON/OFF ratio and a variable spectral response peak (from 900 to $1035 \mathrm{~nm}$ ), enabling bias-control wavelength-sensitive photodetector applications. The GNEC film/n-Si heterojunction photodetector allows low cost, large area, and efficient production. It can be used for a variety of tunable photoelectric devices with high responsivities over broadband, having a broad application potential in industrial production.

Supplementary Materials: The following are available online at http://www.mdpi.com/2079-4991/9/3/327/s1, Figure S1: SEM images of (a) platinum protective layer evaporation and ion etching separation of cross-section, (b) a cross-section sample fabricated by using FIB, and sample located on a copper ring in preparation for TEM observation. (c) Top view of the cross-section, the thickness was thinned to $100 \mathrm{~nm}$ by ion irradiation; Figure S2: Current-voltage (I- $\left.V_{\text {diode }}\right)$ curves under darkness and illumination $(\mathrm{P}=10$ or $40 \mathrm{~mW})$ of $785 \mathrm{~nm}$ laser of carbon films $/ n-S i$ of $V_{\text {dep }}$ at (a) 20V, (b) 50V, and (c) 80V. The GNEC film/n-Si exhibits a large photocurrent under reverse $V_{\text {diode. }}$. As the reverse bias voltage increases from $0 \mathrm{~V}$ to $-2 \mathrm{~V} \sim-3 \mathrm{~V}$, the photocurrent tends to saturate.

Author Contributions: X.Z. and Z.L. conceived and designed the experiments; X.Z., Z.L. and D.P. performed the experiments; X.Z., and Z.L. analyzed the data; X.Z., and D.D. contributed experimental tools; X.Z. and Z.L. wrote the paper, D.D coordinated the project.

Funding: The work was supported by the National Natural Science Foundation (No. 51605306) of China, the Natural Science Foundations of Guangdong (No. 2016A030310060) and Shenzhen (No. 827000131), and the Shenzhen Foundation Fund (No. JCYJ20160427105015701).

Acknowledgments: Thank Wencong Chen for kindly helps. The authors would like to express their appreciation to the Electron Microscope Center (EMC) of Shenzhen University for the instrument time and technical support.

Conflicts of Interest: The authors declare no conflict of interest. 


\section{References}

1. Urban, F.; Peters, L.; Martucciello, N.; Mcevoy, N.; Di Bartolomeo, A. Environmental effects on the electrical characteristics of back-gated WSe ${ }_{2}$ field effect transistors. Nanomaterials 2018, 8, 901. [CrossRef] [PubMed]

2. Xue, Y.Z.; Yuan, J.; Liu, J.Y.; Li, S.J. Controllable Synthesis of 2D Perovskite on Different Substrates and Its Application as Photodetector. Nanomaterials 2018, 8, 591. [CrossRef] [PubMed]

3. Li, X.M.; Zhu, M.; Du, M.D.; Lv, Z.; Zhang, L.; Li, Y.C.; Yang, Y.; Yang, T.T.; Li, X.; Wang, K.L.; et al. High Detectivity Graphene-Silicon Heterojunction Photodetector. Small 2016, 12, 595-601. [CrossRef] [PubMed]

4. Peumans, P.; Yakimov, A.; Forrest, S.R. Small molecular weight organic thin-film photodetectors and solar cells. J. Appl. Phys. 2003, 93, 3693-3723. [CrossRef]

5. Ferrari, A.C. Graphene Photonics and Optoelectronics. Nature Photon. 2016, 4, 611-622.

6. Mueller, T.; Xia, F.; Avouris, P. Graphene photodetectors for high-speed optical communications. Nature Photon. 2010, 4, 297-301. [CrossRef]

7. Zhu, H.; Wei, J.; Wang, K.; Wu, D. Applications of carbon materials in photovoltaic solar cells. Sol. Energy Mater. Sol. Cells. 2009, 93, 1461-1470. [CrossRef]

8. Xie, C.; Mak, C.; Tao, X.; Yan, F. Photodetectors Based on Two-Dimensional Layered Materials Beyond Graphene. Adv. Funct. Mater. 2016, 27. [CrossRef]

9. Yu, H.A.; Kaneko, T.; Otani, S.; Sasaki, Y.; Yoshimura, S. Carbonaceous Thin Film Made by CVD and Its Application for a Carbon/n-Type Silicon (C/n-Si) Photovoltaic Cell. Carbon 1998, 36, 137-143. [CrossRef]

10. Suk, J.W.; Kitt, A.; Magnuson, C.W.; Hao, Y.; Ahmed, S.; An, J.; Swan, A.K.; Goldberg, B.B.; Ruoff, R.S. Transfer of CVD-grown monolayer graphene onto arbitrary substrates. Acs Nano 2011, 5, 6916-6924. [CrossRef] [PubMed]

11. An, Y.; Behnam, A.; Pop, E.; Ural, A. Metal-semiconductor-metal photodetectors based on graphene/p-type silicon Schottky junctions. Appl. Phys. Lett. 2013, 102, 013110. [CrossRef]

12. Yim, C.; Mcevoy, N.; Duesberg, G.S. Characterization of graphene-silicon Schottky barrier diodes using impedance spectroscopy. Appl. Phys. Lett. 2013, 103, 193106. [CrossRef]

13. Ayhan, M.; Kalita, G.; Kondo, M.; Tanemura, M. Photoresponsivity of silver nanoparticles decorated graphene-silicon Schottky junction. Rsc Adv. 2014, 4, 26866-26871. [CrossRef]

14. Sinha, D.; Lee, J.U. Ideal graphene/silicon Schottky junction diodes. Nano Lett. 2014, 14, 4660. [CrossRef] [PubMed]

15. Zhu, M.; Li, X.; Guo, Y.; Li, X.; Sun, P.; Zang, X.; Wang, K.; Zhong, M.; Wu, D.; Zhu, H. Vertical junction photodetectors based on reduced graphene oxide/silicon Schottky diodes. Nanoscale 2014, 6, 4909-4914. [CrossRef] [PubMed]

16. Lu, Y.; Lerner, M.B.; Qi, Z.J.; Mitala, J.J., Jr.; Lim, J.H.; Discher, B.M.; Johnson, A.T.C. Graphene-protein bioelectronic devices with wavelength-dependent photoresponse. Appl. Phys. Lett. 2012, 100, 033110. [CrossRef]

17. Vijapur, S.H.; Wang, D.; Botte, G.G. The growth of transparent amorphous carbon thin films from coal. Carbon 2013, 54, 22-28. [CrossRef]

18. Yu, H.A.; Kaneko, Y.; Yoshimura, S.; Otani, S. Photovoltaic cell of carbonaceous film/n-type silicon. Appl. Phys. Lett. 1998, 68, 547-549. [CrossRef]

19. Krishna, K.M.; Umeno, M.; Nukaya, Y.; Soga, T.; Jimbo, T. Photovoltaic and spectral photoresponse characteristics of $\mathrm{n}-\mathrm{C} / \mathrm{p}-\mathrm{C}$ solar cell on a p-silicon substrate. Appl. Phys. Lett. 2000, 77, 1472-1474. [CrossRef]

20. Xue, Q.; Wang, S.; Li, J.; Zhang, H.; Jiao, Z. Influence of substrate resistivity on photovoltaic characteristics of Pd-doped amorphous carbon film/SiO2/Si heterojunction. Phys. Status Solidi. 2012, 209, 1359-1362. [CrossRef]

21. Ma, M.; Xue, Q.; Li, J.; Zhen, Y.; Wang, S. Light-induced resistance effect of Pd doped carbon film/SiO2/Si. J. Appl. Phys. 2011, 110, 966. [CrossRef]

22. Xue, Q.; Ma, M.; Zhen, Y.; Zhou, X.; Wang, S. Large photoconductivity of Pd doped amorphous carbon film/SiO2/Si. Diamond Relat.Mater. 2012, 21, 24-27. [CrossRef]

23. Wang, C.; Diao, D.; Fan, X.; Chen, C. Graphene sheets embedded carbon film prepared by electron irradiation in electron cyclotron resonance plasma. Appl. Phys. Lett. 2012, 100, 231909. [CrossRef]

24. Yang, L.; Hu, G.; Zhang, D.; Diao, D. Nanosized graphene sheets enhanced photoelectric behavior of carbon film on p-silicon substrate. Appl. Phys. Lett. 2016, 109, 1461-1470. [CrossRef] 
25. Zhang, X.; Zhao, G.; Zhan, L.; Zhan, F.; Wang, J.; Han, G. The effects of microstructure on optical and thermal properties of porous silica films. Surf. Coat. Technol. 2017, 320, 174-177. [CrossRef]

26. Wang, C.; Chen, C.; Diao, D. Top surface modification of carbon film on its structure, morphology and electrical resistivity using electron-ion hybrid irradiation in ECR plasma. Surf. Coat. Technol. 2016, 308, 50-56. [CrossRef]

27. Bogaerts, W.; Wiaux, V.; Taillaert, D.; Beckx, S. Fabrication of photonic crystals in silicon-on-insulator using 248-nm deep UV lithography. IEEE J. Sel. Top. Quantum Electron. 2002, 8, 928-934. [CrossRef]

28. Yoo, S.J.; Kim, C.Y.; Shin, J.W.; Lee, S.G.; Jeong, J.M.; Kim, Y.J.; Lee, S.H.; Kim, J.G. Characterization of an amorphous carbon film covering a Mo grid during in situ heating TEM study. Mater. Charact. 2013, 78, 31-36. [CrossRef]

29. And, S.D.; Munichandraiah, N. Effect of Crystallographic Structure of MnO2 on Its Electrochemical Capacitance Properties. J. Phys. Chem. C 2008, 112, 4406-4417.

30. Quhe, R.; Ma, J.; Zeng, Z.; Tang, K.; Zheng, J.; Wang, Y.; Ni, Z.; Wang, L.; Gao, Z.; Shi, J. Tunable band gap in few-layer graphene by surface adsorption. Sci. Rep. 2013, 3, 1794. [CrossRef]

31. Ferrari, A.C.; Meyer, J.C.; Scardaci, V.; Casiraghi, C.; Lazzeri, M.; Mauri, F.; Piscanec, S.; Jiang, D.; Novoselov, K.S.; Roth, S. Raman spectrum of graphene and graphene layers. Phys. Rev. Lett. 2006, 97, 187401. [CrossRef] [PubMed]

32. Huang, S.M.; Sun, Z.; Lu, Y.F.; Hong, M.H. Ultraviolet and visible Raman spectroscopy characterization of diamond-like carbon film growth by pulsed laser deposition. Appl. Phys. A 2002, 74, 519-523. [CrossRef]

33. Lamberton, R.W.; Morley, S.M.; Maguire, P.D.; McLaughlin, J.A. Monitoring laser-induced microstructural changes of thin film hydrogenated amorphous carbon (a-C: H) using Raman spectroscopy. Thin Solid Films. 1998, 333, 114-125. [CrossRef]

34. Tamor, M.A.; Haire, J.A.; Wu, C.H.; Hass, K.C. Correlation of the optical gaps and Raman spectra of hydrogenated amorphous carbon films. Appl. Phys. Lett. 1989, 54, 123-125. [CrossRef]

35. Zhang, X.; Nie, Y.; Zheng, W.; Kuo, J.-1.; Sun, C.Q. Discriminative generation and hydrogen modulation of the Dirac-Fermi polarons at graphene edges and atomic vacancies. Carbon 2011, 49, 3615-3621. [CrossRef]

36. Liu, X.; Zhang, X.; Bo, M.; Li, L.; Tian, H.; Nie, Y.; Sun, Y.; Xu, S.; Wang, Y.; Zheng, W.; et al. Coordination-Resolved Electron Spectrometrics. Chem. Rev. 2015, 115, 6746-6810. [CrossRef] [PubMed]

37. Fangze, L.; Swastik, K. Quantum Carrier Reinvestment-induced ultrahigh and broadband photocurrent responses in graphene-silicon junctions. Acs Nano 2014, 8, 10270-10279.

38. Xi, Z.; Da, P.; Zezhou, L.; Wencong, C.; Dongfeng, D. Edge Effect on the Photodetection Ability of the Graphene Nanocrystallites Embedded Carbon Film Coated on p-Silicon. Phys. Status Solidi RRL 2019, 1800511.

39. Zhang, B.Y.; Liu, T.; Meng, B.; Li, X.; Liang, G.; Hu, X.; Wang, Q.J. Broadband high photoresponse from pure monolayer graphene photodetector. Nat. Commun. 2013, 4, 1811. [CrossRef] [PubMed]

40. Yu, Y.; Kats, M.A.; Raji, S.; Yi, S.; Jing, K.; Marko, L.; Federico, C. Wide wavelength tuning of optical antennas on graphene with nanosecond response time. Nano Lett. 2014, 14, 214-219.

41. Wu, W.; Zhang, Q.; Zhou, X.; Li, L.; Su, J.; Wang, F.; Zhai, T. Self-powered photovoltaic photodetector established on lateral monolayer MoS2-WS2 heterostructures. Nano Energy. 2018, 51, 45-53. [CrossRef]

42. Liu, Y.; Cheng, R.; Liao, L.; Zhou, H.; Bai, J.; Liu, G.; Liu, L.; Huang, Y.; Duan, X. Plasmon resonance enhanced multicolour photodetection by graphene. Nat. Commun. 2011, 2, 579. [CrossRef] [PubMed]

43. Woo Jong, Y.; Yuan, L.; Hailong, Z.; Anxiang, Y.; Zheng, L.; Yu, H.; Xiangfeng, D. Highly efficient gate-tunable photocurrent generation in vertical heterostructures of layered materials. Nat. Nanotech. 2013, 8, 952-958.

44. Lv, P.; Zhang, X.; Zhang, X.; Deng, W.; Jie, J. High-Sensitivity and Fast-Response Graphene/Crystalline Silicon Schottky Junction-Based Near-IR Photodetectors. IEEE Electron Device Lett. 2013, 34, 1337-1339. [CrossRef]

45. Zhang, X.; Wang, C.; Sun, C.Q.; Diao, D.F. Magnetism induced by excess electrons trapped at diamagnetic edge-quantum well in multi-layer graphene. Appl. Phys. Lett. 2014, 105, 042402. [CrossRef]

46. An, X.; Liu, F.; Jung, Y.J.; Kar, S. Tunable Graphene-Silicon Heterojunctions for Ultrasensitive Photodetection. Nano Lett. 2013, 13, 909-916. [CrossRef] [PubMed]

(C) 2019 by the authors. Licensee MDPI, Basel, Switzerland. This article is an open access article distributed under the terms and conditions of the Creative Commons Attribution (CC BY) license (http:/ / creativecommons.org/licenses/by/4.0/). 